\title{
User Experience at Work: Four Perspectives on What It May Mean
}

\author{
Morten Hertzum \\ University of Copenhagen, Copenhagen, Denmark \\ hertzum@hum.ku.dk
}

\begin{abstract}
Most work involves the use of artifacts; thus, user experience (UX) is a factor in how most employees experience their work. This study revisits the tool, media, dialogue-partner, and system perspectives on artifact use to explore UX at work. It is found that artifacts foster positive UX when they lend the user expressive power (tool), are transparent (media) or perceptive (dialogue partner). They foster negative UX when they attract the user's attention or make the user a mere system component. The task focus inherent in the perspectives suggests that wellbeing at work is mostly promoted by factors other than UX.
\end{abstract}

\section{Keywords}

Perspectives on artifact use · User experience $\cdot$ Wellbeing · Work

\section{Introduction}

User experience (UX) is about the experiences associated with the use of artifacts [12]. Some definitions restrict UX to actual use [2], others include anticipated use [9], and still others also include aesthetics [5]. These differences appear, however, minor compared to the shared focus on the experiences associated

How to cite this book chapter:

Hertzum, M. 2020. User Experience at Work: Four Perspectives on What It May Mean. In: Loizides, F., Winckler, M., Chatterjee, U., Abdelnour-Nocera, J. and Parmaxi, A. (eds.) Human Computer Interaction and Emerging Technologies: Adjunct Proceedings from the INTERACT 2019 Workshops. Pp. 127-132. Cardiff: Cardiff University Press. DOI: https://doi.org/10.18573/book3.o. License: CC-BY 4.0. 
with artifact use. Well-documented experiences with computer artifacts in work settings include burnout, deskilling, frustration, and helplessness [e.g., 4, 11]. Countering such negative experiences is central to employee wellbeing; replacing them with positive experiences would be an even nobler design goal.

Studies of technology acceptance find that perceived enjoyment, a concept similar to UX, predicts the intention to use an artifact as strongly as do perceived usefulness and perceived ease of use [8]. The relation between the artifact and the user's experience is, however, complex because UX is not determined by the artifact alone. Rather, UX results from the interrelations among the characteristics of the artifact, user, task, and context of use. While this observation is largely trivial, it raises the question of whether UX exerts much influence on wellbeing at work. It may well be that wellbeing at work is first and foremost facilitated by factors other than computer artifacts, that is, by experiences and conditions other than UX.

To explore what we might accomplish by designing for good UX at work this study revisits Kammersgaard's [10] four perspectives on human-computer interaction, ponders what constitutes positive and negative UX within each perspective, and discusses possible positive contributions of UX to wellbeing at work.

\section{Four Perspectives on System Use and UX}

Kammersgaard [10] outlines four perspectives on human-computer interaction by distinguishing between artifacts for individual and collaborative use and between artifacts for which agency rests with the user and artifacts that split agency between user and artifact. The four perspectives are the tool perspective, the system perspective, the dialogue-partner perspective, and the media perspective, see Table 1 .

Table 1: Four perspectives on system use, adapted from Kammersgaard [10].

\begin{tabular}{|c|c|}
\hline Individual & Collaborative \\
\hline User agency Tool perspective & Media perspective \\
\hline $\begin{array}{l}\text { - Artifact is an extension of } \\
\text { the user's body }\end{array}$ & $\begin{array}{l}\text { - Users communicate through the } \\
\text { artifact }\end{array}$ \\
\hline $\begin{array}{l}\text { - Ready to hand vs present } \\
\text { at hand }\end{array}$ & - Media richness vs common ground \\
\hline - UX?: expressive power & - UX?: transparency, structure \\
\hline Split agency Dialogue-partner perspective & System perspective \\
\hline $\begin{array}{l}\text { - Artifact displays human- } \\
\text { like behavior }\end{array}$ & $\begin{array}{l}\text { - User is similar to other system } \\
\text { components }\end{array}$ \\
\hline $\begin{array}{l}\text { - Intelligent vs annoying } \\
\text { assistant }\end{array}$ & - Automation vs meaningful jobs \\
\hline - UX?: perceptive, adaptive & - UX?: deskilling, monotony \\
\hline
\end{tabular}


The tool perspective has its roots in craftwork and emphasizes that in the hands of a skilled user the tool is a seamless extension of the user, who attends to her task rather than to the tool: When hammering the skilled user's attention is on driving the nail, not on the hammer. Conceptually, the tool is said to be ready to hand [14]. It is only upon breakdowns that the tool becomes the focus of the user's attention - becomes present at hand. If the hammer is too light for the size of nail or otherwise inadequate for the task then the user's attention shifts from the task to the tool. These shifts are associated with frustration and other negative emotions because the breakdown thwarts progress on the task, at least temporarily. It appears that tools foster good experiences when they are out of mind - ready to hand - and poor experiences when they become present at hand. If we take the definition of UX to mean that the user must, in the moment, be conscious that she is using an artifact then the tool perspective rules out positive UX. The positive experiences do not qualify as UX because they are associated with an uninterrupted focus on the task. In contrast, the user is conscious of the artifact when it thwarts task progress; thus tools can foster negative UX. If we do not require that the user must, in the moment, be conscious that she is using an artifact - and this is probably the more sensible option - then positive UX is possible within the tool perspective and consists of designing for readiness to hand. The user may however not attribute the positive UX to the tool but, partly or wholly, to other aspects of the use situation.

The system perspective aligns with industrial perceptions of work and promotes a view in which a system consists of components that may be human or automated. Each component is characterized by the input it receives, the activities it performs on those inputs, and the outputs it delivers. The division of the system into components is made by management and defines a division of labor. To perform their work the users need only know the characteristics of the component they embody. Performance is measured by how cheaply, quickly, and consistently the components deliver their outputs. That is, the users' work is measured in the same way as that of the automated components. If the users perform poorer than an automated version of the same component then the users are at risk of being replaced by such a component. In this sense the users are measured by their ability to function as automated components. The automation inherent in the system perspective is often associated with deskilling of the users, who become operators of machines that perform more and more components of the work [1]. This negative UX results from a primary focus on automation, thereby leaving the users with the components that have not yet been automated. To create positive UX it is necessary to focus on creating meaningful and rewarding human components, for example by automating the parts of work that are monotonous or unhealthy. However, to create meaningful and rewarding human components it may also be necessary to reconsider the separation between a managerial level that defines the components and an operational level that merely performs according to these preset definitions. That is, it may be necessary to challenge the essence of the system perspective. 
The dialogue-partner perspective sees the artifact as an intelligent assistant with which the users can interact in much the same way as they interact with humans. The intelligent assistant empowers the user by serving his or her needs and does so without requiring that the user learns special commands for interacting with the assistant. Unlike the system perspective, which tends to reduce humans to machines, the dialogue-partner perspective seeks to elevate machines to human-like performance. Unlike the tool perspective, which involves the user's moment-to-moment handling of the tool, the intelligent assistant acts autonomously in the user's service. The intelligent assistant may, for example, monitor an architect's work on a building and inform the architect when his current building design violates formal regulations or recognized principles for good design [3]. The intelligent assistant fosters positive and negative UX in much the same way as a human collaborator. Negative UX ensues if the assistant needs too many instructions, performs poor work, or delivers its work at inopportune moments. Positive UX ensues if the assistant is effective and efficient and, especially, if the assistant also picks up on the tacit conditions for good performance and reacts appropriately to dynamic changes in the environment. Often, intelligent assistants must be supervised by users who need to be ready to take over if the assistant encounters a situation it cannot handle. This creates poor conditions for positive UX because the user wants to offload the task to the assistant but must, instead, "stay in the loop" to be ready to step in whenever needed.

The media perspective positions the artifact as a medium through which the users interact with each other. That is, the medium is merely a channel; agency rests with the users. Rich media [13] provide for simultaneous interactions in multiple modalities and, thereby, for back-channeling (e.g., nods, raised eyebrows) to occur via some modalities at the same time as the main interaction occupies other modalities (e.g., speech). Thereby, rich media support users in establishing, sustaining, and repairing common ground, which is key to effective collaborative interactions. Conversely, lean media provide few or only a single modality and may be restricted to asynchronous interactions, thereby increasing the risk of breakdowns in common ground. Media provide positive UX when they are transparent - somewhat similar to when a tool is ready to hand. A transparent medium allows the interactions among the users to flow without distortions. Rich media are transparent with respect to more interaction modalities than lean media. In addition to transparency, some media aim to provide positive UX by structuring the interaction, for example by making explicit that an interactional turn is a request and therefore must be answered by accepting, declining or negotiating the request [14]. Media foster negative UX when they are insufficiently transparent or enforce a structure that is too rigid. In both cases the medium stands in the way of the interactions among the users. 


\section{Discussion}

Most work involves the use of artifacts, such as products, systems or services. Thus, UX is a factor in how most employees experience their work. In the tool and media perspectives, an artifact fosters positive UX by not attracting the user's attention, which instead remains on the task. That is, it is by supporting the user in expressing her skills - as manifested in high-quality work task products - that tools and media foster positive UX. Positive UX is about lending the user expressive power. In the dialogue-partner perspective, positive UX is as much about how well the artifact engages in the process of its use as it is about the product that results from this process. That is, an artefact fosters positive UX if it is a perceptive and adaptive dialogue partner. In the system perspective, positive UX appears to be secondary to other concerns. That is, positive UX involves abandoning the system perspective or, at least, supplementing it with other perspectives. Abandoning the system perspective is a daunting undertaking because this perspective permeates much thinking about how to organize workplaces. For example, physicians are increasingly frustrated that they spend still more of their time documenting their work in electronic patient records and comparatively less time with patients, but the increasing documentation requirements are justified by pointing out that the physician is a component in a much larger system, which needs the documentation for hospital-level quality assurance, national performance indicators, and international healthcare research [4].

A less ambitious goal than fostering positive UX in the service of wellbeing at work would be to avoid negative UX. The tool and media perspectives agree that artifacts foster negative UX whenever they attract the user's attention. Thus, users become conscious of their artifact use when they experience problems with the artifacts. The distinction between, on the one hand, positive UX and a task focus and, on the other hand, negative UX and an artifact focus echoes a seminal study of wellbeing at work. In this study Herzberg et al. [7, p. 113] conclude:

When our respondents reported feeling happy with their jobs, they most frequently described factors related to their tasks, to events that indicated to them that they were successful in the performance of their work, and to the possibility of professional growth. Conversely, when feelings of unhappiness were reported, they were not associated with the job itself but with conditions that surround the doing of the job.

It may seem that factors other than the use of artifacts stand a better chance of creating wellbeing and that UX may mostly be about avoiding negative experiences. Finally, influence on the design of the artifacts used in performing the work may be a source of positive UX not covered by the four perspectives revisited in this study [6]. 


\section{References}

1. Braverman, H.: Labor and monopoly capital: The degradation of work in the twentieth century. Monthly Review Press, New York (1974).

2. Colbert, M.: User experience of communication before and during rendezvous: Interim results. Personal and Ubiquitous Computing 9(3), 134-141 (2005).

3. Fischer, G., Lemke, A.C., Mastaglio, T.: Critics: An emerging approach to knowledge-based human-computer interaction. International Journal of Man-Machine Studies 35(5), 695-721 (1991).

4. Gawande, A.: The upgrade: Why doctors hate their computers. The New Yorker, 62-73 (November 12, 2018).

5. Hekkert, P.: Design aesthetics: Principles of pleasure in product design. Psychology Science 48(2), 157-172 (2006).

6. Hertzum, M., Torkilsheyggi, A.: How do users perceive a design-in-use approach to implementation? A healthcare case. In: Proceedings of the INTERACT2019 Conference on Human-Computer Interaction. Springer, Cham (2019).

7. Herzberg, F., Mausner, B., Snyderman, B.B.: The motivation to work. Wiley, New York (1959).

8. Hornbæk, K., Hertzum, M.: Technology acceptance and user experience: A review of the experiential component in HCI. ACM Transactions on Computer-Human Interaction 24(5), Article 33 (2017).

9. ISO 9241: Ergonomics of human-system interaction - Part 210: Human-centred design for interactive systems. International Standard Organization, Geneva, CH (2010).

10. Kammersgaard, J.: Four different perspectives on human-computer interaction. International Journal of Man-Machine Studies 28(4), 343-362 (1988).

11. Lazar, J., Jones, A., Shneiderman, B.: Workplace user frustration with computers: An exploratory investigation of the causes and severity. Behaviour \& Information Technology 25(3), 239-251 (2006).

12. Roto, V., Law, E., Vermeeren, A., Hoonhout, J.: User experience white paper: Bringing clarity to the concept of user experience. Result from Dagstuhl Seminar (2011). http://www.allaboutux.org/uxwhitepaper (accessed April 1, 2019).

13. Trevino, L.K., Lengel, R.H., Daft, R.L.: Media symbolism, media richness, and media choice in organizations: A symbolic interactionist perspective. Communication Research 14(5), 553-574 (1987).

14. Winograd, T., Flores, F.: Understanding computers and cognition: A new foundation for design. Ablex, Norwood, NJ (1986). 